Scientific Visualization, 2021, volume 13, number 4, pages 76 - 92, DOI: 10.26583/sv.13.4.07

\title{
Methods of Constructing a Visual Map of Generalized Computational Experiment
}

\author{
A.A. Zakharova1,A,B, D.A. Korostelyov²,A,C, A.G. Podvesovskii3,A,C, V.A. Galaktionov4,A \\ A Keldysh Institute of Applied Mathematics RAS \\ B Institute of Control Sciences RAS \\ C Bryansk State Technical University
}

${ }^{1}$ ORCID: 0ooo-0003-4221-7710, zaawmail@gmail.com

2 ORCID: 0000-0002-0853-7940,nigm85@mail.ru

3 ORCID: 000o-0002-1118-3266, apodv@tu-bryansk.ru

4 ORCID: 0000-0001-6460-7539, vlgal@gin.keldysh.ru

\begin{abstract}
The paper considers the problem of evaluating the state of a generalized computational experiment in the context of a general problem of creating methods for adaptive planning and control of a generalized computational experiment in mathematical modeling of real physical processes. A generalized computational experiment implies multiple solution of the numerical simulation problem for various sets of values of defining model parameters. As a method for assessing a generalized computational experiment state, it is proposed to visualize a set of experimental data specifying this state then followed by analysis of the resulting visual image. An approach to visualization of a generalized computational experiment state is proposed based on constructing a visual map. The concept of a visual map of a generalized computational experiment is introduced, and several methods of its construction are proposed. Examples of application of those methods are considered when assessing the accuracy of numerical models of the OpenFOAM software platform for a three-dimensional problem of inviscid flow around a cone.
\end{abstract}

Keywords: generalized computational experiment, generalized computational experiment state, multidimensional data, visualization, visual analytics, visual map, approximation, problem of flow around a cone, OpenFOAM.

\section{Introduction}

In the tasks of mathematical modeling of physical processes a computational experiment plays an important role. In the general case it consists in a series of calculations with varying the defining parameters of the model. In this case, the task of the experiment is often to simultaneously investigate the influence of several parameters on the characteristics of modeling object of interest, including investigating their joint influence in various combinations of variation ranges. With the development of computer technology, it became possible to construct a so-called generalized computational experiment (GCE) [1], which assumes parallel calculation of the same problem with different sets of parameter values in multitask mode. Currently, there are examples of successful construction and application of a GCE in solving problems of computational fluid dynamics [1-2], gas dynamics [3-4], power plant design automation [5].

From the point of view of representing the results, a generalized computational experiment is characterized by a multidimensional array the elements of which specify the distribution of values of simulation output parameters for a given set of input parameters. In this case, some generalized indicators often act as output parameters, which are the results of processing primary experimental data, contain information about general patterns and 
relationships inherent in the object of modeling, and are used for interpretation, search for patterns, formation and testing of hypotheses. Examples of generalized indicators are the principal components in dimension reduction problems [6], L1 and L2 error vector norms in problems of estimating the accuracy of various numerical methods with varying the key simulation parameters [3-4], etc.

It is obvious that both conducting a GCE and processing and interpreting its results are very resource-intensive tasks. Moreover, it is not possible to conduct an experiment with all allowable combinations of models and simulation parameters. Therefore, it is necessary to resort to GCE planning choosing a specific scenario for its implementation. At the same time, it is advisable to build a GCE on the basis of not a static, predetermined, but a dynamic, adaptively changing plan. The principle of constructing such a plan can be as follows: based on the results of a series of experiments for a certain set of values of input parameters and processing of its results in conjunction with the results of previous series of experiments, the current GCE state is recorded. This state must be evaluated and analyzed in order to determine or adjust the plan for the subsequent series of experiments. To do this, it is necessary to establish dependencies of the output simulation parameters on the input ones and on this basis to select the value ranges of the input parameters for which more detailed studies are required with new or refined sets of parameters. Such situations may arise, for example, when new patterns are discovered that require confirmation and refinement, or if the results of some already conducted experiments do not correspond to the expected patterns and thus require rechecking.

As a method for evaluating a GCE state, it is proposed to visualize the experimental data that define it, followed by analysis of the resulting visual image. The approach based on visualization of multidimensional data has proven itself well in the tasks of exploratory analysis and hypothesis formation [7]. Visual analysis of a GCE state helps to visually and quickly enough detect and highlight problematic or promising value ranges of input parameters that are subject to more detailed study.

A number of papers are devoted to the study of visualization problems in a generalized computational experiment, among which, for example, $[2,3,5]$ can be noted. At the same time, these studies are mainly aimed at application of visualization methods for the analysis and interpretation of experimental results. Visualization methods that could be used to evaluate a GCE state in order to clarify the scenario for its implementation are currently out of consideration. This paper attempts to fill this gap. The concept of a visual map of a generalized computational experiment is introduced, which is understood as a set of visual images characterizing the state of GCE and arranged in accordance with certain rules. Several methods are proposed for constructing a visual map of the GCE, the application of which is considered by the example of evaluating the accuracy of numerical models of the OpenFOAM software platform [8] for a three-dimensional problem of inviscid flow around a cone.

\section{The Concept of Visual Map of Generalized Computational Experiment}

Earlier in the article [9], the authors considered the problem of adaptive planning and control of a generalized computational experiment and proposed the general structure of the GCE control model. It was shown that the key concept for this model is the concept of the state of a generalized computational experiment. The state of the GCE is determined by a set of computational experiments already carried out and is set by a multidimensional array of experimental data obtained with the current partition of the space of defining parameters, a set of generalized indicators obtained as a result of processing this array, as well as a set of patterns identified on the basis of analysis and interpretation of these indicators.

With regard to the problem of constructing a visual image of the GCE, the following formal representation of the state of the GCE can be proposed, which can be considered as a refinement and concretization of the formal representation proposed in [9] in the context of the visualization problem. 
Let us assume that within the framework of a GCE on a set of models $M=\left\{m_{1}, m_{2}, \ldots\right.$, $m_{N m}$ ), where $N m$ is the number of models, $N k$ computational experiments were carried out within which the input simulation parameters $P=\left\{p_{1}, p_{2}, \ldots, p_{N p}\right\}$ were varied, where $N p$ is the number of input simulation parameters. As a result of the computational experiments, the output parameters $S=\left\{s_{1}, s_{2}, \ldots, s_{N s}\right\}$ were determined, where $N s$ is the number of output parameters. As noted above, the output parameters can be generalized indicators, which are the results of processing the primary experimental data. Each computational experiment $k$ was carried out for a fixed set of input simulation parameters $P_{k}=\left\{p_{k, 1}, p_{k, 2}, \ldots, p_{k, N k}\right\}$. In this case, situations are possible when, for a fixed set of simulation parameters, computational experiments were not performed on all models. The results of the performed computational experiments form a set $R=\left\{r_{k, m, v}\right\}$, where $k$ is the number of the computational experiment $(1 \leq k \leq N k), m$ is the number of the model $(1 \leq m \leq N m), v$ is the number of the output parameter $(1 \leq v \leq N s)$.

Thus, the state of the GCE is determined by a given partition of the space of values of the input parameters of modeling from the set $P$, the set of values of the output parameters included in the set $S$, as well as the results of a series of computational experiments that form the set $R$.

The visual image of the state of the GCE can be presented in the form of a set of visual elements on a plane or in space, linked in a certain way with each other. Each visual element reflects a subset of the set $R$ of GCE results and is characterized by a set of visual features. In this work, we will restrict ourselves to considering the following visual signs:

- coordinates on the plane $(x, y)$ or in space $(x, y, z)$;

- Shape;

- Size;

- Color.

At the same time, we note that in addition to the listed features, it is possible to use others, such as color saturation, orientation, texture, etc.

With a large number of modeling parameters, a separate visual image of the state of the GCE is able to reflect only a certain part of the set $R$ of its results. Thus, to visualize the state of the GCE, it is necessary to construct a set of interconnected visual images. We will call such set a visual map of a generalized computational experiment.

To construct a visual map of the GCE, you first need to prepare the data, which consists in transforming the initial representation of the state of the GCE to a set of dependencies of the following type.

For the two-dimensional case:

For the three-dimensional case:

$$
\begin{gathered}
F(x)=<y, \text { Shape, Size, Color }>, \\
\text { or } F(x, y)=<\text { Shape, Size, Color }>
\end{gathered}
$$

$$
\begin{gathered}
F(x)=<y, z \text {, Shape, Size, Color }>, \\
\text { or } F(x, y)=<z \text {, Shape, Size, Color }>, \\
\text { or } F(x, y, z)=<\text { Shape, Size, Color }>.
\end{gathered}
$$

In this case, the arguments of the function $F$ are determined primarily by the input parameters of the modeling, and the sets of its values are primarily associated with the output parameters, as well as with the characteristics of the experimental results for which the visual image is constructed. Some methods for such a transformation are proposed in this work and will be discussed in the next section.

The resulting set of visual images can be subjected to preliminary analysis using wellknown methods and visual analytics tools. This approach assumes that each visual image is analyzed separately, with subsequent comparison and integration of the results of such analysis. We will call this approach interactive.

To obtain a holistic visual presentation, a variety of visual images are converted into a visual map, which in particular involves the search for a suitable transformation method, for example, in the form of a certain set of rules for layout visual images. The approach to the visual analysis of the state of GCE based on the construction of a visual map will be called a complex one. 


\section{Methods of Data Preparation for Constructing a Visual Map of Generalized Computational Experiment}

Let us visualize the state of the GCE using a series of two-dimensional approximating curves of the results of computational experiments. Considering the fact that there can be several input simulation parameters, let us apply the following algorithm (Fig. 1) to construct two-dimensional approximating curves for one model $m$.

1. Fix sequentially each input parameter $p_{i}$. Values of this parameter determine the values of $x$ pairs $(x ; y)$ for which we will further carry out approximation.

2. Determine set $T=\left\{t_{d}\right\}$ of all possible combinations of the remaining input parameters $p_{j}\left(p \neq p_{i}\right)$, where $1 \leq d \leq N t_{i}, N t_{i}$ is the number of possible combinations of the remaining input parameters for a fixed parameter $p_{i}$.

3. For each such combination $t_{d}$, obtain $N s_{d}$ dependences of the output parameters $s_{v}$ on the parameter $p_{i}$ (it is assumed that not all combinations of input values could be obtained for all output parameters).

4. Carry out construction of the functional dependence using approximating functions. To do this, first determine the number - Nfi,d, and the form of possible approximating functions $F_{i, d, v}=\left\{f_{i, d, v, 1}, f_{i, d, v, 2}, \ldots, f_{i, d, v, N f_{i, d, v}}\right\}$ for each resulting parameter $s_{v}$, a combination of the input parameters $d$ and a fixed input parameter $i$. The values of the resulting parameters $s_{v}$ (the corresponding $r_{k, m, v}$ are selected from the set $R$ ) specify the values of $y$ in pairs $(x ; y)$ for which we will carry out the approximation.

5. For each approximating function $f_{i, d, v, j}$ for the resulting parameter $s_{v}$ and each combination $t_{d}$ of input parameters, a graph of functional dependence on the parameter $p_{i}$ construct and approximation accuracy is determined $e_{i, d, v, j}$.

6. Visually compare of graph shapes is carried out for a fixed parameter $p_{i}$ for different approximating functions, and deviations and patterns are revealed. Among other things, shapes of the curves obtained for different resulting parameters are compared.

7. Choose the following fixed input parameter: $i=i+1$ and go to step 2 .

8. If all the input parameters are exhausted, then the algorithm is completed.

The presented algorithm is repeated for each model.

The method based on application of this algorithm makes it possible to visually identify deviations in the results of experiments and determine patterns; however, it does not reflect a GCE state as a whole, since it forms not a single visual image but a series of visual images. In other words, this method implements an interactive approach to visual analysis of the GCE state and can be considered as an intermediate stage in the process of constructing a visual GCE map.

For the transition from a series of visual images to a single visual map, it is proposed to use the following method. The obtained characteristics of approximation accuracy $e_{i, t, v, j}$ are summarized in tables: for each pair of a fixed input parameter $i$ and an output parameter $v$, we obtain one table, the columns of which are approximating functions $f_{j, v}$, and the rows are specific values of the remaining parameters. The cells of this table are the corresponding values of approximation accuracy $-e_{i, t, v, j}$. For each row of these tables, let us define characterizing values by the following methods:

1. Minimum accuracy of approximation (one parameter).

2. Maximum accuracy of approximation (one parameter).

3. Average accuracy of approximation and root-mean-square deviation of different methods of approximation from the mean (two parameters). 


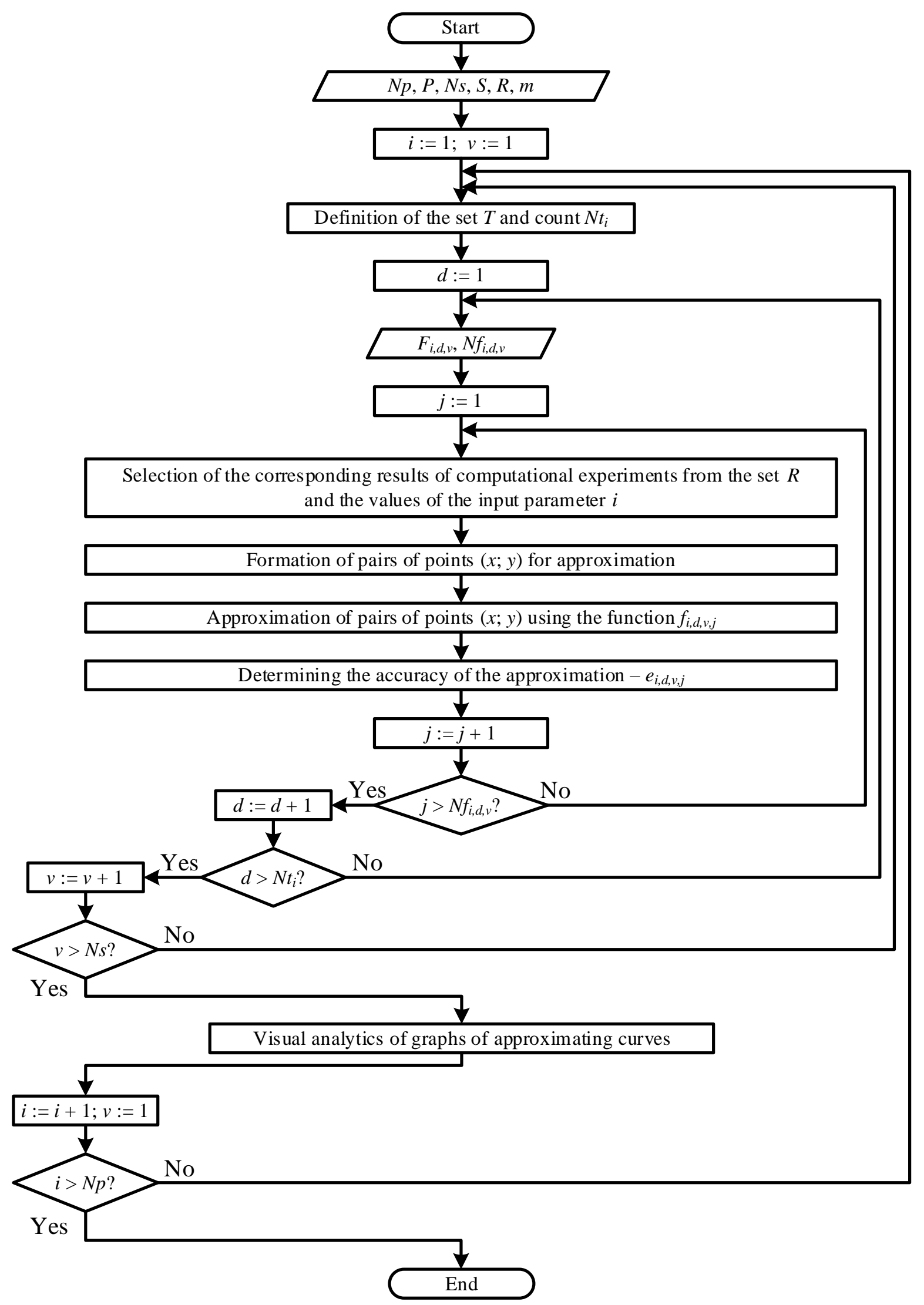

Fig. 1. Flowchart of visualization algorithm for a GCE state using a series of approximating graphs

Visualization of the obtained characteristics will be carried out using two-dimensional dot plots. On this graph, for each resulting parameter $v$, the corresponding points will be of the same color. For different resulting parameters $v$, the colors will be different. We will also visualize different input parameters $i$ using different colors (different types of markers can be also used). The ordinal number of the table rows will be used as the value of the point along 
the abscissa axis in a two-dimensional visualization. The characterizing values will be used as values along the ordinate axis. When using the 3 rd method for determining the characteristic values, the average accuracy will determine the value along the ordinate axis, and the rootmean-square deviation will determine the size of the point.

As a result, a set of dot plots will again be obtained, but their number is already significantly less than in the previous approach, and various layout methods can be applied to this set of visual images, such as overlay, horizontal and vertical alignment, etc. At the same time, to give the resulting image more visual expressiveness, you can additionally use various types of transformation of coordinate grids - stretching, rotation, mirroring.

Thus, on one two-dimensional dot plot, we can reflect a GCE state for one specific model. Analyzing the relative position (and the size of the points for the third method of determining the characteristic values) of the points, it is possible to visually determine for which input parameters there are problems with the approximation accuracy, and as a consequence, errors in carrying out computational experiments are possible, and for which it is not yet possible to determine the best approximation methods and, consequently, determine the value ranges of the input parameters for which it makes sense to carry out additional computational experiments in intermediate values.

\section{Experiment Description}

Let us consider application of the proposed methods of constructing a visual map for a GCE carried out within evaluating the accuracy of solvers of the OpenFOAM platform (in OpenFOAM terminology, solvers are software modules in which various numerical models of mechanics of continua are implemented [8]) for the three-dimensional problem of inviscid flow around a cone [4, 10] (Fig. 2). Solvers rhoCentralFoam, pisoCentralFoam, sonicFoam will be considered as models $M$.

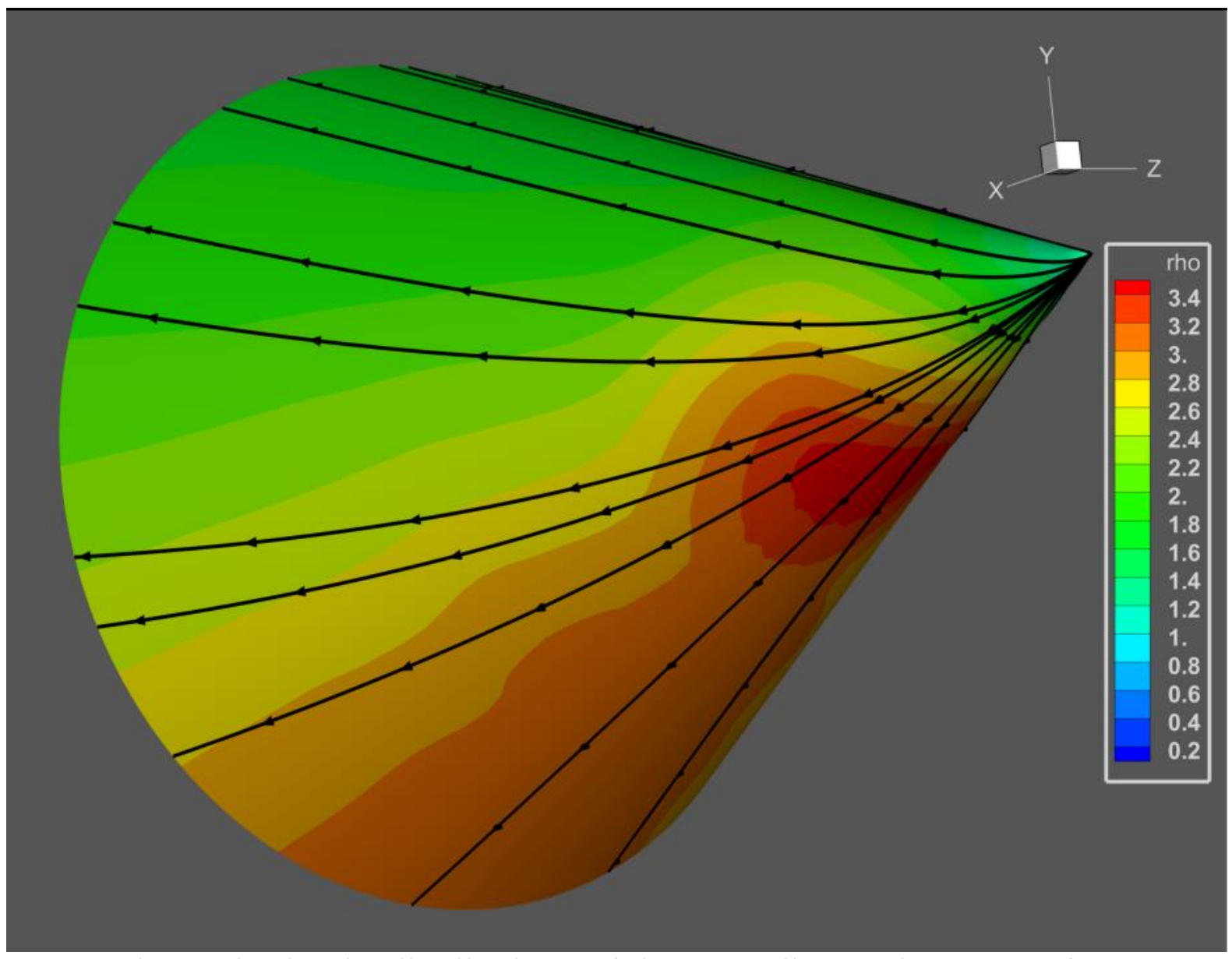

Fig. 2. The density distribution and the streamline on the cone surface in a supersonic flow angle of attack 
The simulation input parameters $(P)$ are Mach number $(M a)$, cone half-angle (Betta, in degrees), and angle of attack (Angle, in degrees). The output parameters (S) of computational experiments are: the results of calculating deviation norms L1 and L2 of the numerical calculation from the analytical solution.

As approximating functions, we will use the following:

- linear $(y=a x+b)$;

- exponential $\left(y=a e^{b x}\right)$;

- logarithmic $(y=a \ln (x)+b$;

- quadratic $\left(y=a x^{2}+b x+c\right)$. [11].

As the approximation accuracy, we will use the value of the approximation reliability $R^{2}$

As a result of the approximation of the obtained data, 178 approximating functions were constructed for each solver, considering the fact that some types of approximations in specific cases could not be carried out. For example, for the quadratic approximation, in the case of only two points, the graph was expressed in a line and therefore was not taken into account. Also, for the logarithmic function, it was impossible to determine approximating functions for the cases when the value of the parameter $x$ could be equal to o. The visualization results of the obtained approximating functions are partially shown in Fig. 3-5. For convenience, one graph displays the curves for all output parameters (norms L1 and L2).
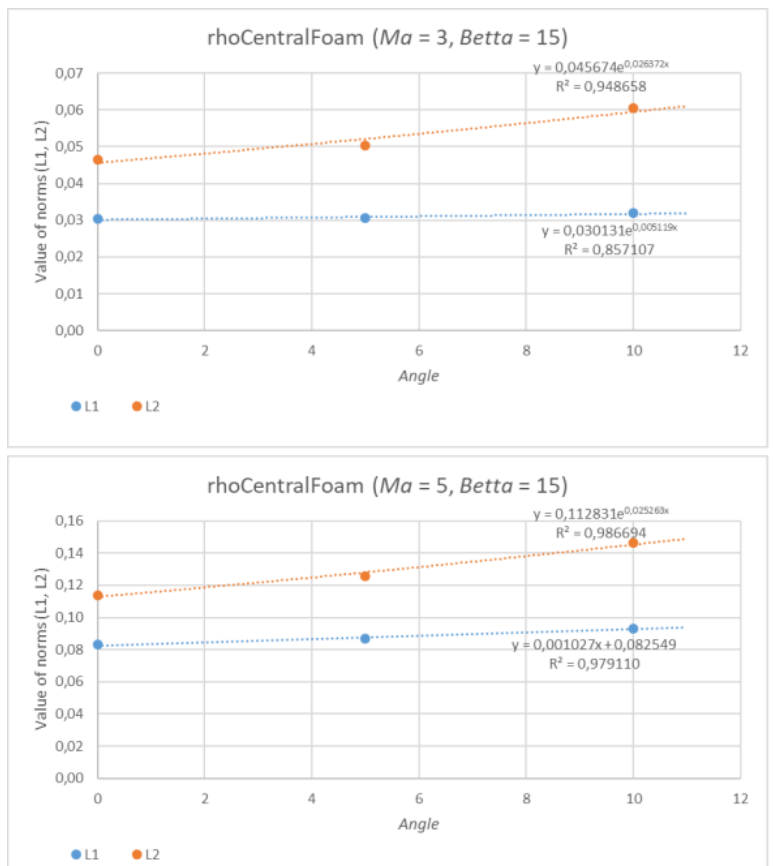

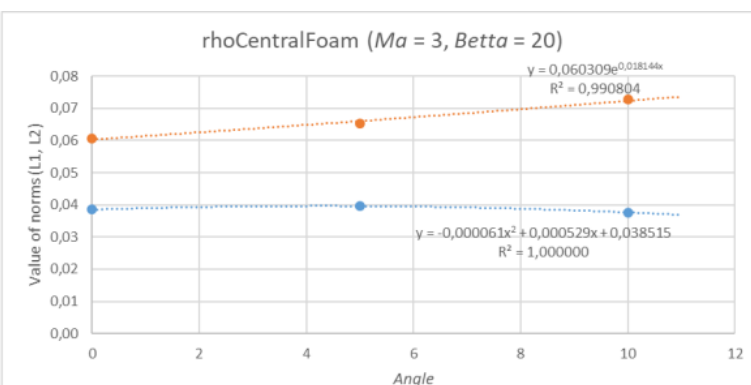

$\bullet$ เ1 เ

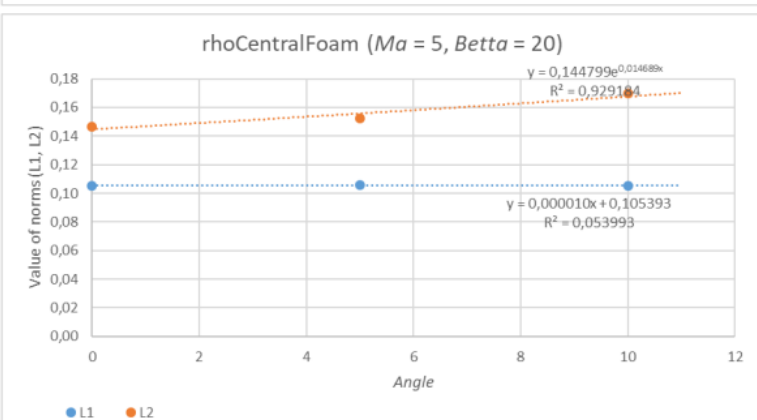

Fig. 3. An example of visualization of approximating functions for rhoCentralFoam solver with a fixed input parameter - angle of attack 

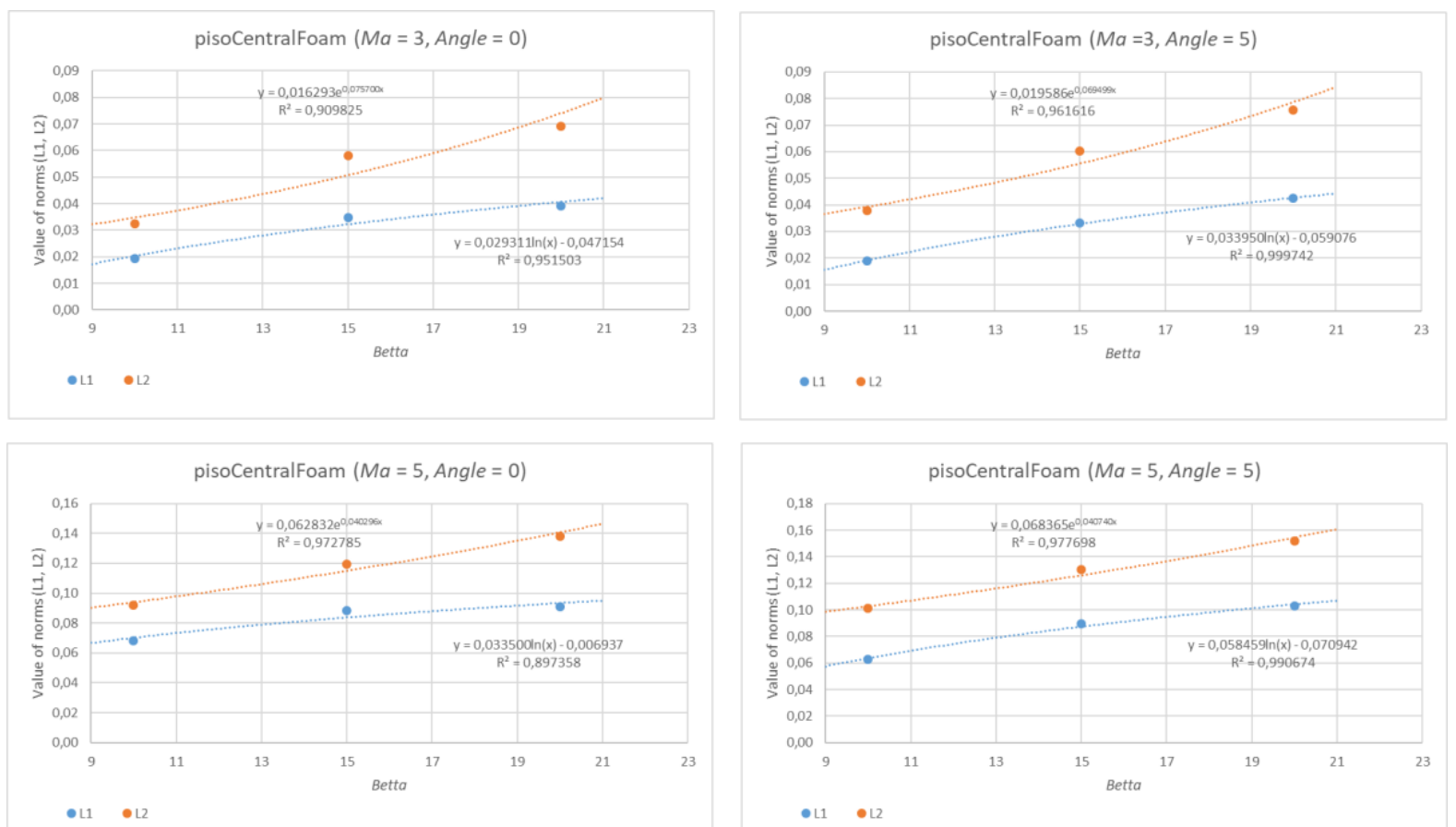

Fig. 4. An example of visualization of approximating functions for psioCentralFoam solver with a fixed input parameter - cone half-angle
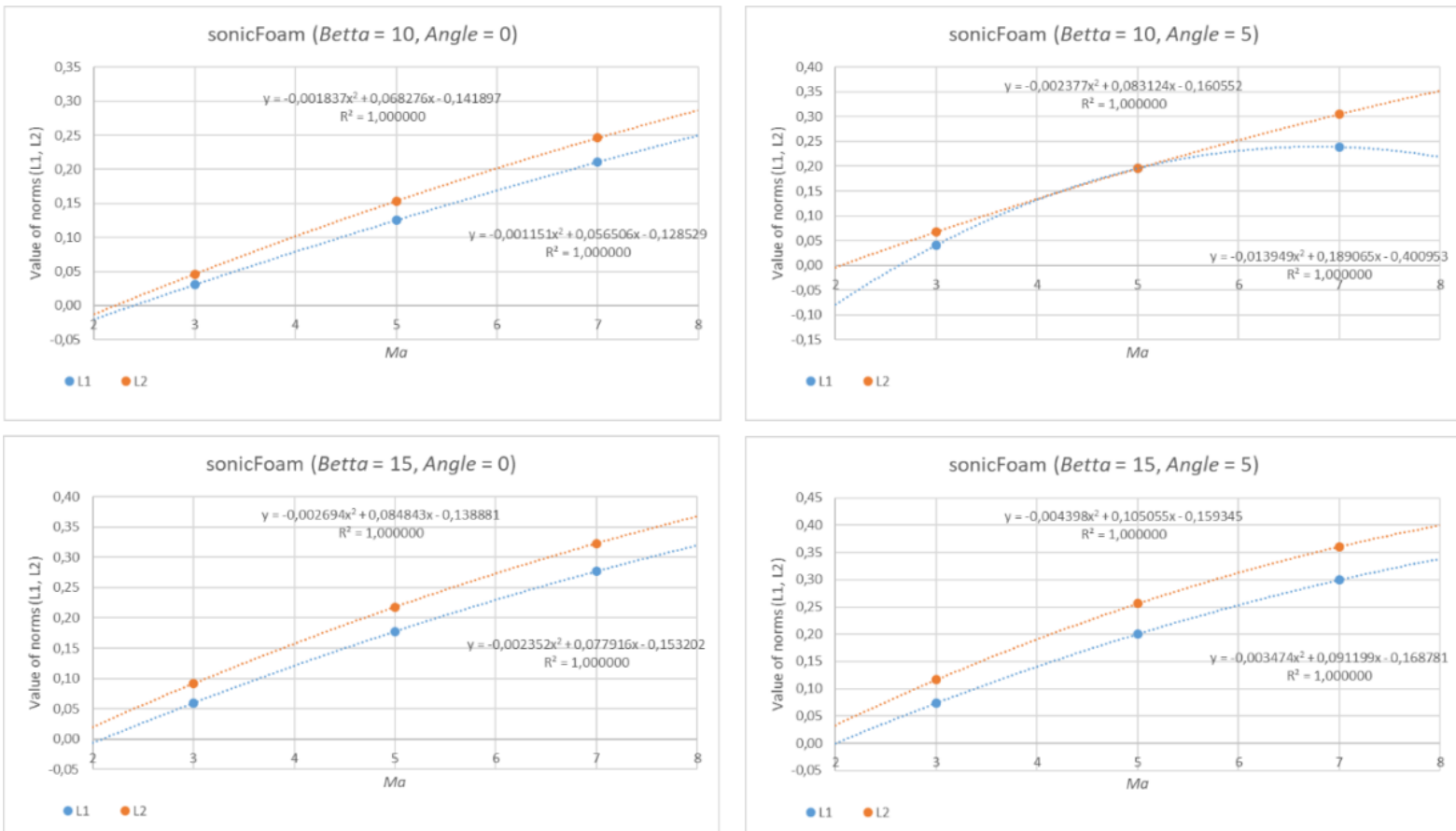

Fig. 5. An example of visualization of approximating functions for sonicFoam solver with a fixed input parameter - Mach number

After constructing a series of diagrams and determining the approximation accuracy, their mean values and root-mean-square deviations were calculated (Tables 1-9). Bubble charts were used for visual map constructing, herewith a different bubble color means belonging to different data series (a combination of a fixed input parameter and an output parameter), and the size of a point (bubble) characterizes the root-mean-square deviation of the approximation accuracy by different types of curves. Fig. 6-8 show thus obtained visual map of GCE state for different solvers. The map consists of three bubble charts, each one of which summarizes the results of evaluating the accuracy of the corresponding solver by representing them as a single visual image. 
Table 1. Characterizing values of approximation for rhoCentralFoam solver for Ma fixed parameter

\begin{tabular}{|c|c|c|c|c|c|c|c|c|c|c|}
\hline \multirow{2}{*}{$\begin{array}{c}\text { Input } \\
\text { parameters } \\
\text { combination } \\
\text { values }\end{array}$} & \multirow{2}{*}{ Comb. number } & Min & Max & Avg & RMSD & Min & Max & Avg & RMSD \\
\cline { 1 - 9 } & & & & & & & & & & \\
\hline 10 & 0 & 1 & 0,94309 & 1 & 0,97756 & 0,00177 & 0,96078 & 1 & 0,97931 & 0,00078 \\
\hline 10 & 5 & 2 & 0,94501 & 1 & 0,97771 & 0,00164 & 0,96456 & 1 & 0,97964 & 0,00067 \\
\hline 15 & 0 & 3 & 0,95181 & 1 & 0,98145 & 0,00130 & 0,96382 & 1 & 0,98245 & 0,00066 \\
\hline 15 & 5 & 4 & 0,95938 & 1 & 0,98295 & 0,00096 & 0,96914 & 1 & 0,98295 & 0,00049 \\
\hline 15 & 10 & 5 & 0,96687 & 1 & 0,98423 & 0,00078 & 0,97589 & 1 & 0,98439 & 0,00037 \\
\hline 20 & 0 & 6 & 0,97009 & 1 & 0,98661 & 0,00069 & 0,97306 & 1 & 0,98468 & 0,00038 \\
\hline 20 & 5 & 7 & 0,97103 & 1 & 0,98600 & 0,00065 & 0,97530 & 1 & 0,98478 & 0,00035 \\
\hline 20 & 10 & 8 & 0,95286 & 1 & 0,98151 & 0,00124 & 0,96410 & 1 & 0,98312 & 0,00065 \\
\hline
\end{tabular}

Table 2. Characterizing values of approximation for rhoCentralFoam solver for Betta fixed parameter

\begin{tabular}{|c|c|c|c|c|c|c|c|c|c|c|}
\hline \multicolumn{2}{|c|}{$\begin{array}{c}\text { Input } \\
\text { parameters } \\
\text { combination } \\
\text { values }\end{array}$} & \multirow{2}{*}{ Comb. number } & Min & Max & Avg & RMSD & Min & Max & Avg & RMSD \\
\cline { 1 - 11 } Ma & Angle & & & & & & & & & \\
\hline 3 & 0 & 1 & 0,95043 & 1 & 0,98336 & 0,00161 & 0,95677 & 1 & 0,98652 & 0,00126 \\
\hline 3 & 5 & 2 & 0,95835 & 1 & 0,98699 & 0,00117 & 0,96930 & 1 & 0,99076 & 0,00063 \\
\hline 3 & 10 & 3 & 1 & 1 & 1 & 0 & 1 & 1 & 1 & 0 \\
\hline 5 & 0 & 4 & 0,97991 & 1 & 0,99376 & 0,00026 & 0,98595 & 1 & 0,99512 & 0,00012 \\
\hline 5 & 5 & 5 & 0,96365 & 1 & 0,98749 & 0,00087 & 0,98076 & 1 & 0,99376 & 0,00024 \\
\hline 5 & 10 & 6 & 1 & 1 & 1 & 0 & 1 & 1 & 1 & 0 \\
\hline 7 & 0 & 7 & 0,94123 & 1 & 0,97376 & 0,00216 & 0,97109 & 1 & 0,98996 & 0,00056 \\
\hline 7 & 5 & 8 & 0,95094 & 1 & 0,97905 & 0,00154 & 0,97709 & 1 & 0,99178 & 0,00035 \\
\hline 7 & 10 & 9 & 1 & 1 & 1 & 0 & 1 & 1 & 1 & 0 \\
\hline
\end{tabular}

Table 3. Characterizing values of approximation for rhoCentralFoam solver for Angle fixed parameter

\begin{tabular}{|c|c|c|c|c|c|c|c|c|c|c|}
\hline \multirow{2}{*}{\multicolumn{2}{|c|}{$\begin{array}{c}\text { Input } \\
\text { parameters } \\
\text { combination } \\
\text { values } \\
\end{array}$}} & \multirow{3}{*}{ Comb. number } & \multicolumn{4}{|c|}{ L1 } & \multicolumn{4}{|c|}{ L2 } \\
\hline & & & \multirow[t]{2}{*}{ Min } & \multirow[t]{2}{*}{$\operatorname{Max}$} & \multirow[t]{2}{*}{ Avg } & \multirow[t]{2}{*}{ RMSD } & \multirow[t]{2}{*}{ Min } & \multirow[t]{2}{*}{$\operatorname{Max}$} & \multirow[t]{2}{*}{ Avg } & \multirow[t]{2}{*}{ RMSD } \\
\hline Ma & Betta & & & & & & & & & \\
\hline 3 & 10 & 1 & 1 & 1 & 1 & o & 1 & 1 & 1 & o \\
\hline 3 & 15 & 2 & 0,85490 & 1 & 0,90400 & 0,01383 & 0,93488 & 1 & 0,96118 & 0,00236 \\
\hline 3 & 20 & 3 & 0,18749 & 1 & 0,46021 & 0,43708 & 0,98542 & 1 & 0,99207 & 0,00011 \\
\hline 5 & 10 & 4 & 1 & 1 & 1 & o & 1 & 1 & 1 & o \\
\hline 5 & 15 & 5 & 0,97911 & 1 & 0,98748 & 0,00024 & o,97777 & 1 & 0,98816 & 0,00025 \\
\hline 5 & 20 & 6 & o,05399 & 1 & 0,36938 & 0,59651 & 0,92132 & 1 & 0,95017 & o,00376 \\
\hline 7 & 10 & 7 & 1 & 1 & 1 & o & 1 & 1 & 1 & o \\
\hline 7 & 15 & 8 & 0,96513 & 1 & 0,97780 & 0,00074 & 0,99184 & 1 & 0,99594 & 0,00003 \\
\hline 7 & 20 & 9 & 0,91134 & 1 & 0,94293 & 0,00490 & 0,91572 & 1 & 0,94759 & 0,00418 \\
\hline
\end{tabular}


Table 4. Characterizing values of approximation for pisoCentralFoam solver for Ma fixed parameter

\begin{tabular}{|c|c|c|c|c|c|c|c|c|c|c|}
\hline \multirow{2}{*}{\multicolumn{2}{|c|}{$\begin{array}{c}\text { Input } \\
\text { parameters } \\
\text { combination } \\
\text { values } \\
\end{array}$}} & \multirow{3}{*}{ Comb. number } & \multicolumn{4}{|c|}{$\mathbf{L 1}$} & \multicolumn{4}{|c|}{ L2 } \\
\hline & & & \multirow[t]{2}{*}{ Min } & \multirow[t]{2}{*}{$\operatorname{Max}$} & \multirow[t]{2}{*}{ Avg } & \multirow[t]{2}{*}{ RMSD } & \multirow[t]{2}{*}{ Min } & \multirow[t]{2}{*}{$\operatorname{Max}$} & \multirow[t]{2}{*}{ Avg } & \multirow[t]{2}{*}{ RMSD } \\
\hline Betta & Angle & & & & & & & & & \\
\hline 10 & o & 1 & 0,96572 & 1 & 0,98198 & 0,00102 & 0,96733 & 1 & 0,98194 & 0,00055 \\
\hline 10 & 5 & 2 & 0,95111 & 1 & 0,97941 & 0,00132 & 0,97196 & 1 & 0,98231 & 0,00047 \\
\hline 15 & o & 3 & 0,96671 & 1 & 0,98576 & 0,00069 & 0,96975 & 1 & 0,98576 & 0,00046 \\
\hline 15 & 5 & 4 & 0,97213 & 1 & 0,98645 & 0,00066 & 0,97739 & 1 & 0,98607 & 0,00030 \\
\hline 15 & 10 & 5 & 0,97034 & 1 & 0,98600 & 0,00071 & 0,97381 & 1 & 0,98663 & 0,00034 \\
\hline 20 & o & 6 & 0,97305 & 1 & 0,98788 & 0,00052 & 0,98156 & 1 & 0,98771 & 0,00023 \\
\hline 20 & 5 & 7 & 0,97348 & 1 & 0,98835 & 0,00054 & 0,97972 & 1 & 0,98775 & 0,00023 \\
\hline 20 & 10 & 8 & 0,96188 & 1 & 0,98500 & 0,00084 & 0,96349 & 1 & 0,98492 & 0,00073 \\
\hline
\end{tabular}

Table 5. Characterizing values of approximation for pisoCentralFoam solver for Betta fixed parameter

\begin{tabular}{|c|c|c|c|c|c|c|c|c|c|c|}
\hline \multirow{2}{*}{\multicolumn{2}{|c|}{$\begin{array}{c}\text { Input } \\
\text { parameters } \\
\text { combination } \\
\text { values }\end{array}$}} & \multirow{3}{*}{ Comb. number } & \multicolumn{4}{|c|}{ L1 } & \multicolumn{4}{|c|}{ L2 } \\
\hline & & & \multirow[t]{2}{*}{ Min } & \multirow[t]{2}{*}{ Max } & \multirow[t]{2}{*}{ Avg } & \multirow[t]{2}{*}{ RMSD } & \multirow[t]{2}{*}{ Min } & \multirow[t]{2}{*}{$\operatorname{Max}$} & \multirow[t]{2}{*}{ Avg } & \multirow[t]{2}{*}{ RMSD } \\
\hline Ma & Angle & & & & & & & & & \\
\hline 3 & O & 1 & 0,86753 & 1 & 0,93004 & 0,01010 & 0,90982 & 1 & 0,96023 & 0,00477 \\
\hline 3 & 5 & 2 & 0,95472 & 1 & 0,98539 & 0,00136 & 0,96162 & 1 & 0,98745 & 0,00098 \\
\hline 3 & 10 & 3 & 1 & 1 & 1 & o & 1 & 1 & 1 & O \\
\hline 5 & $\mathrm{O}$ & 4 & 0,82152 & 1 & 0,88742 & 0,02032 & 0,97279 & 1 & 0,98998 & 0,00050 \\
\hline 5 & 5 & 5 & 0,93841 & 1 & 0,97293 & 0,00235 & 0,97770 & 1 & 0,99217 & 0,00033 \\
\hline 5 & 10 & 6 & 1 & 1 & 1 & o & 1 & 1 & 1 & $\mathrm{O}$ \\
\hline 7 & o & 7 & 0,61822 & 1 & 0,73936 & 0,09566 & 0,94428 & 1 & 0,97283 & 0,00200 \\
\hline 7 & 5 & 8 & 0,94427 & 1 & 0,97283 & 0,00200 & 0,97481 & 1 & 0,98972 & 0,00045 \\
\hline 7 & 10 & 9 & 1 & 1 & 1 & O & 1 & 1 & 1 & O \\
\hline
\end{tabular}

Table 6. Characterizing values of approximation for pisoCentralFoam solver for Angle fixed parameter

\begin{tabular}{|c|c|c|c|c|c|c|c|c|c|c|}
\hline \multirow{2}{*}{\multicolumn{2}{|c|}{$\begin{array}{c}\text { Input } \\
\text { parameters } \\
\text { combination } \\
\text { values } \\
\end{array}$}} & \multirow{3}{*}{ Comb. number } & \multicolumn{4}{|c|}{ L1 } & \multicolumn{4}{|c|}{ L2 } \\
\hline & & & \multirow[t]{2}{*}{ Min } & \multirow[t]{2}{*}{$\operatorname{Max}$} & \multirow[t]{2}{*}{ Avg } & \multirow[t]{2}{*}{ RMSD } & \multirow[t]{2}{*}{ Min } & \multirow[t]{2}{*}{$\operatorname{Max}$} & \multirow[t]{2}{*}{ Avg } & \multirow[t]{2}{*}{ RMSD } \\
\hline Ma & Betta & & & & & & & & & \\
\hline 3 & 10 & 1 & 1 & 1 & 1 & o & 1 & 1 & 1 & o \\
\hline 3 & 15 & 2 & 0,91702 & 1 & 0,94593 & 0,00439 & 0,87596 & 1 & 0,92025 & 0,00958 \\
\hline 3 & 20 & 3 & 0,10339 & 1 & 0,40438 & 0,53217 & 0,99539 & 1 & 0,99778 & 0,00001 \\
\hline 5 & 10 & 4 & 1 & 1 & 1 & o & 1 & 1 & 1 & o \\
\hline 5 & 15 & 5 & 0,98832 & 1 & 0,99256 & 0,00008 & 0,97153 & 1 & 0,98409 & 0,00042 \\
\hline 5 & 20 & 6 & 0,44460 & 1 & 0,63462 & 0,20036 & 0,98909 & 1 & 0,99411 & 0,00006 \\
\hline 7 & 10 & 7 & 1 & 1 & 1 & o & 1 & 1 & 1 & o \\
\hline 7 & 15 & 8 & 0,59444 & 1 & 0,73036 & 0,10906 & 0,98688 & 1 & o,99292 & 0,00009 \\
\hline 7 & 20 & 9 & 0,95681 & 1 & 0,97358 & 0,00107 & 0,97079 & 1 & 0,98366 & 0,00044 \\
\hline
\end{tabular}


Table 7. Characterizing values of approximation for sonicFoam solver for Ma fixed parameter

\begin{tabular}{|c|c|c|c|c|c|c|c|c|c|c|}
\hline \multirow{2}{*}{\multicolumn{2}{|c|}{$\begin{array}{c}\text { Input } \\
\text { parameters } \\
\text { combination } \\
\text { values } \\
\end{array}$}} & \multirow{3}{*}{ Comb. number } & \multicolumn{4}{|c|}{$\mathbf{L 1}$} & \multicolumn{4}{|c|}{ L2 } \\
\hline & & & \multirow[t]{2}{*}{ Min } & \multirow[t]{2}{*}{$\operatorname{Max}$} & \multirow[t]{2}{*}{ Avg } & \multirow[t]{2}{*}{ RMSD } & \multirow[t]{2}{*}{ Min } & \multirow[t]{2}{*}{ Max } & \multirow[t]{2}{*}{ Avg } & \multirow[t]{2}{*}{ RMSD } \\
\hline Betta & Angle & & & & & & & & & \\
\hline 10 & $\mathrm{o}$ & 1 & 0,93393 & 1 & 0,98130 & 0,00303 & 0,94069 & 1 & 0,98329 & 0,00244 \\
\hline 10 & 5 & 2 & 0,83358 & 1 & 0,92504 & 0,01578 & 0,94642 & 1 & 0,98478 & 0,00197 \\
\hline 15 & o & 3 & 0,94378 & 1 & 0,98416 & 0,00218 & 0,95478 & 1 & 0,98693 & o,00139 \\
\hline 15 & 5 & 4 & 0,94249 & 1 & 0,98381 & 0,00229 & 0,95059 & 1 & 0,98562 & 0,00166 \\
\hline 15 & 10 & 5 & 0,95377 & 1 & 0,98653 & 0,00145 & 0,95639 & 1 & 0,98714 & 0,00128 \\
\hline 20 & $\mathrm{o}$ & 6 & 0,94410 & 1 & 0,98423 & 0,00216 & 0,95462 & 1 & 0,98681 & 0,00140 \\
\hline 20 & 5 & 7 & 0,95508 & 1 & 0,98701 & 0,00137 & 0,96682 & 1 & 0,98993 & 0,00072 \\
\hline 20 & 10 & 8 & 0,94389 & 1 & 0,98367 & 0,00216 & 0,93180 & 1 & 0,97619 & 0,00303 \\
\hline
\end{tabular}

Table 8. Characterizing values of approximation for sonicFoam solver for Betta fixed parameter

\begin{tabular}{|c|c|c|c|c|c|c|c|c|c|c|}
\hline \multirow{2}{*}{\multicolumn{2}{|c|}{$\begin{array}{c}\text { Input } \\
\text { parameters } \\
\text { combination } \\
\text { values } \\
\end{array}$}} & \multirow{3}{*}{ Comb. number } & \multicolumn{4}{|c|}{$\mathbf{L 1}$} & \multicolumn{4}{|c|}{ L2 } \\
\hline & & & \multirow[t]{2}{*}{ Min } & \multirow[t]{2}{*}{ Max } & \multirow[t]{2}{*}{ Avg } & \multirow[t]{2}{*}{ RMSD } & \multirow[t]{2}{*}{ Min } & \multirow[t]{2}{*}{ Max } & \multirow[t]{2}{*}{ Avg } & \multirow[t]{2}{*}{ RMSD } \\
\hline Ma & Angle & & & & & & & & & \\
\hline 3 & o & 1 & 0,95646 & 1 & 0,98717 & 0,00130 & 0,95243 & 1 & 0,98572 & 0,00154 \\
\hline 3 & 5 & 2 & 0,95830 & 1 & 0,98732 & 0,00118 & 0,95756 & 1 & 0,98654 & 0,00120 \\
\hline 3 & 10 & 3 & 1 & 1 & 1 & o & 1 & 1 & 1 & O \\
\hline 5 & 0 & 4 & 0,97308 & 1 & 0,99112 & 0,00048 & 0,97513 & 1 & 0,99194 & 0,00041 \\
\hline 5 & 5 & 5 & 0,77291 & 1 & 0,86953 & 0,02695 & 0,95457 & 1 & 0,98051 & 0,00134 \\
\hline 5 & 10 & 6 & 1 & 1 & 1 & o & 1 & 1 & 1 & o \\
\hline 7 & o & 7 & 0,97176 & 1 & 0,98968 & 0,00054 & 0,97402 & 1 & 0,99071 & 0,00045 \\
\hline 7 & 5 & 8 & 0,97764 & 1 & 0,99180 & 0,00034 & 0,98942 & 1 & 0,99606 & 0,00007 \\
\hline 7 & 10 & 9 & 1 & 1 & 1 & o & 1 & 1 & 1 & O \\
\hline
\end{tabular}

Table 9. Characterizing values of approximation for sonicFoam solver for Angle fixed parameter

\begin{tabular}{|c|c|c|c|c|c|c|c|c|c|c|}
\hline \multirow{2}{*}{\multicolumn{2}{|c|}{$\begin{array}{c}\text { Input } \\
\text { parameters } \\
\text { combination } \\
\text { values } \\
\end{array}$}} & \multirow{3}{*}{ Comb. number } & \multicolumn{4}{|c|}{ L1 } & \multicolumn{4}{|c|}{ L2 } \\
\hline & & & \multirow[t]{2}{*}{ Min } & \multirow[t]{2}{*}{$\operatorname{Max}$} & \multirow[t]{2}{*}{ Avg } & \multirow[t]{2}{*}{ RMSD } & \multirow[t]{2}{*}{ Min } & \multirow[t]{2}{*}{$\operatorname{Max}$} & \multirow[t]{2}{*}{ Avg } & \multirow[t]{2}{*}{ RMSD } \\
\hline $\mathbf{M a}$ & Betta & & & & & & & & & \\
\hline 3 & 10 & 1 & 1 & 1 & 1 & $\mathrm{O}$ & 1 & 1 & 1 & $\mathrm{O}$ \\
\hline 3 & 15 & 2 & 0,96358 & 1 & 0,98025 & 0,00068 & 0,99746 & 1 & 0,99906 & o \\
\hline 3 & 20 & 3 & 0,89731 & 1 & 0,93560 & 0,00630 & 0,99195 & 1 & 0,99672 & 0,00004 \\
\hline 5 & 10 & 4 & 1 & 1 & 1 & $\mathrm{O}$ & 1 & 1 & 1 & $\mathrm{O}$ \\
\hline 5 & 15 & 5 & 0,96283 & 1 & o,97789 & 0,00077 & 0,99861 & 1 & 0,99953 & $\mathrm{O}$ \\
\hline 5 & 20 & 6 & 0,97636 & 1 & 0,98561 & 0,00032 & 0,95714 & 1 & 0,97520 & 0,00099 \\
\hline 7 & 10 & 7 & 1 & 1 & 1 & O & 1 & 1 & 1 & O \\
\hline 7 & 15 & 8 & 0,99736 & 1 & o,99886 & $\mathrm{O}$ & 0,99584 & 1 & 0,99829 & 0,00001 \\
\hline 7 & 20 & 9 & 0,66821 & 1 & 0,77960 & 0,07287 & 0,99866 & 1 & 0,99946 & O \\
\hline
\end{tabular}

When constructing visual maps in Fig. 6-8, the overlay layout method was used. This made it possible to reflect several different combinations of the values of the input and output parameters in one diagram. If you need to focus on each combination separately, then you can use graph alignment as a layout method (table layout: left column - L1 norm, right 
column - L2 norm, rows - fixed input parameter). As a result, we get a visual map consisting of three sets of graphs shown in Fig. 9-11.

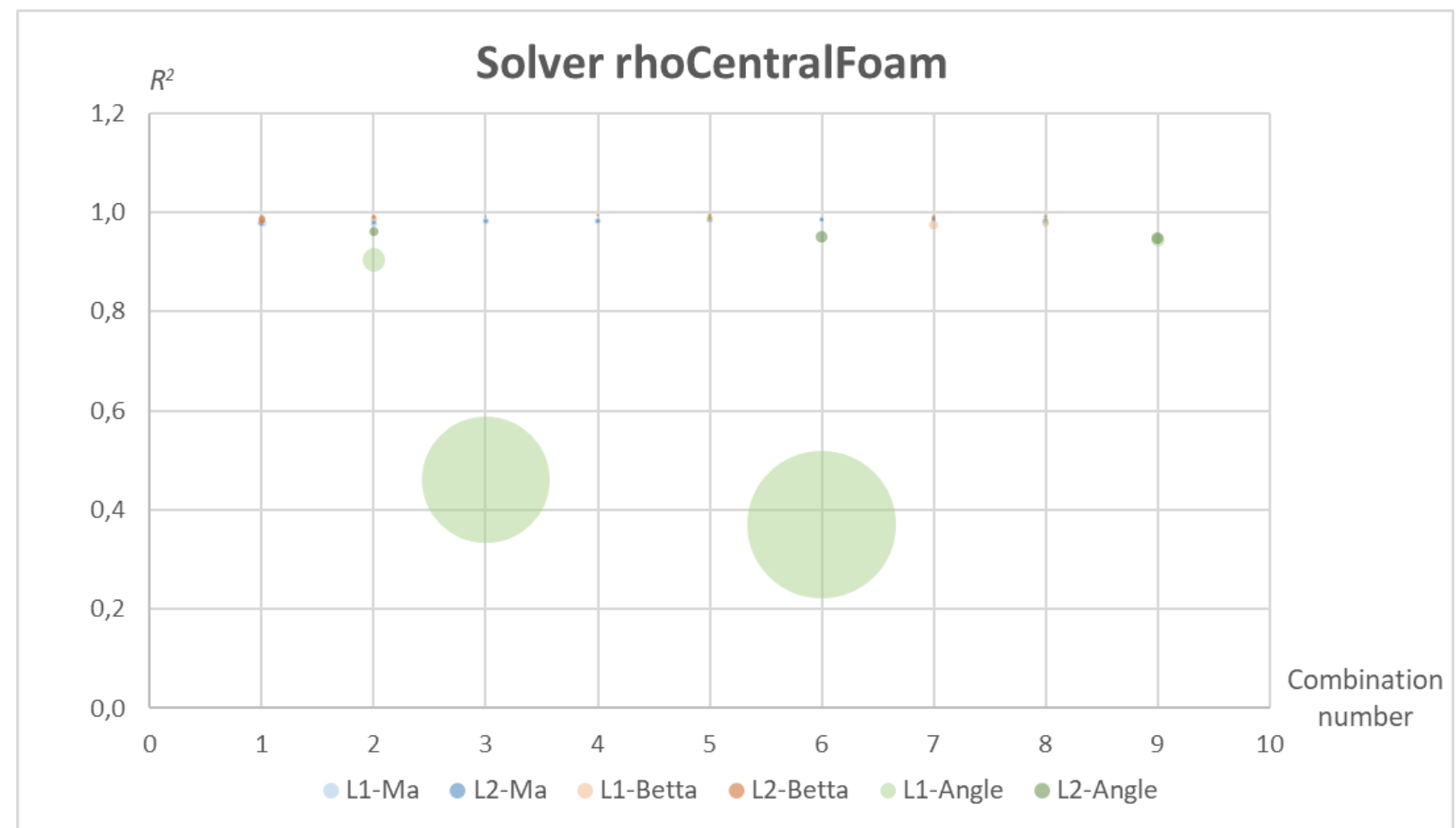

Fig. 6. Visual map of the GCE for rhoCentralFoam solver (overlay method)

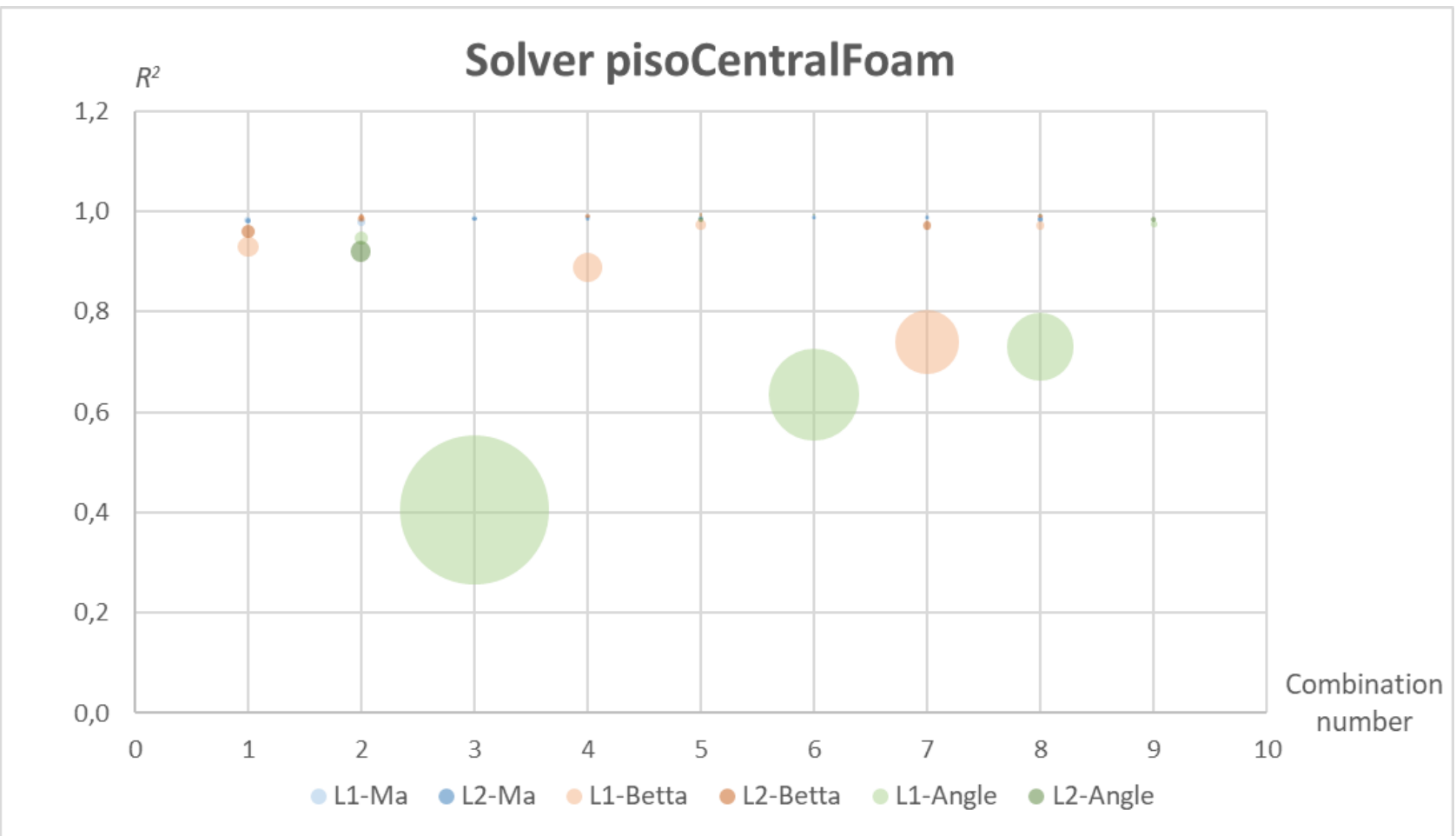

Fig. 7. Visual map of the GCE for pisoCentralFoam solver (overlay method) 


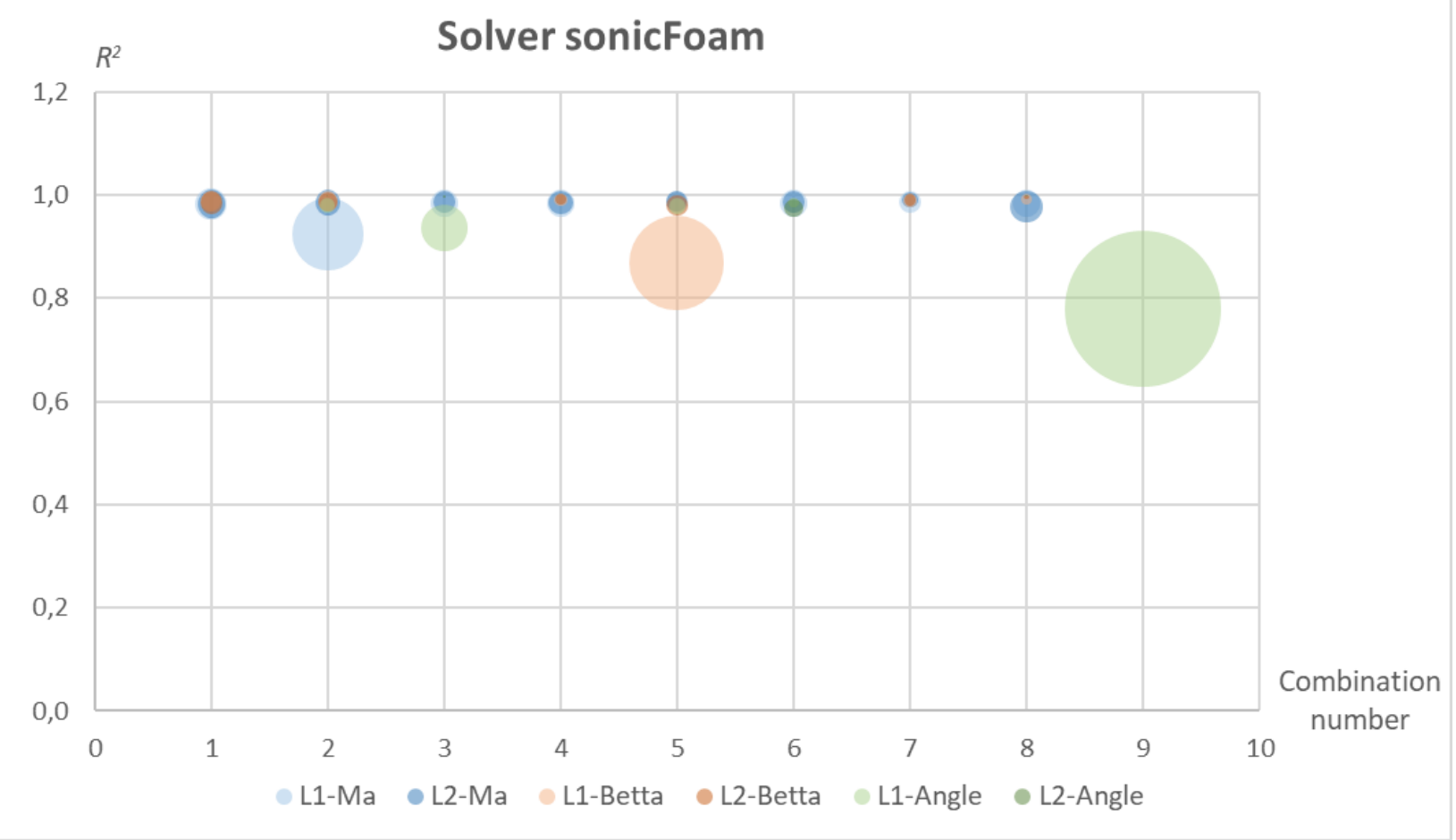

Fig. 8. Visual map of the GCE for sonicFoam solver (overlay method)
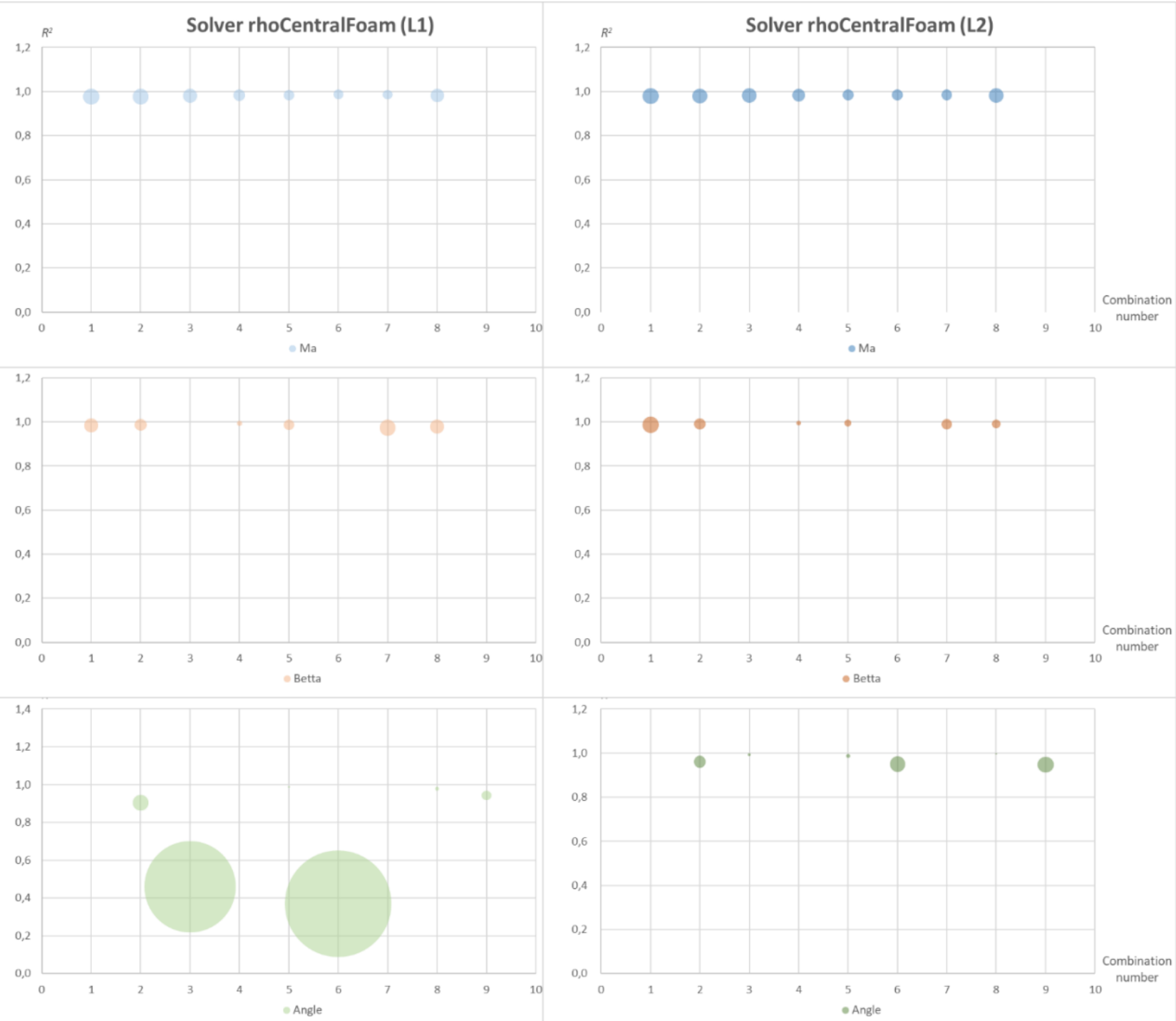

Fig. 9. Visual map of the GCE for rhoCentralFoam solver (table layout) 


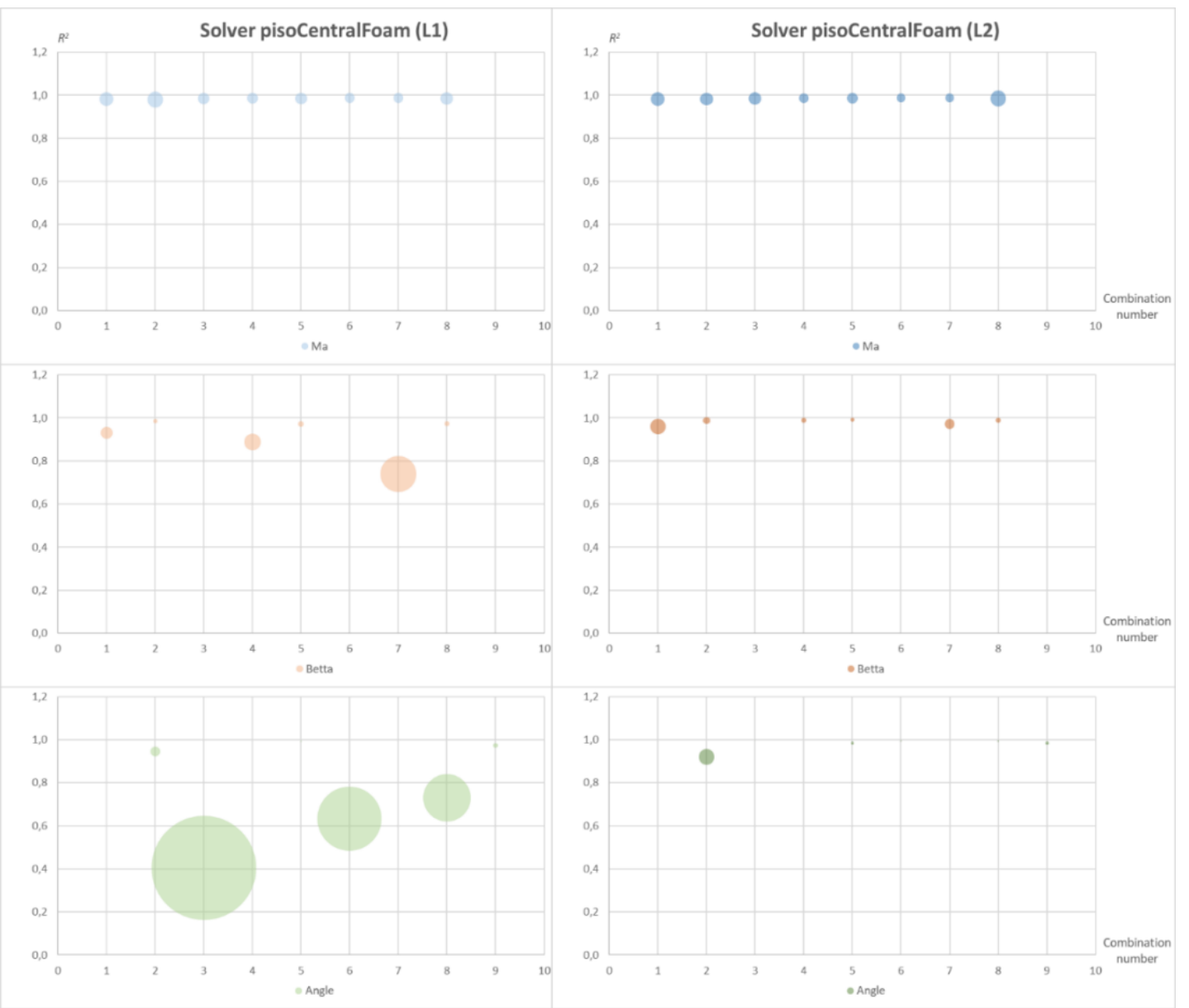

Fig. 10. Visual map of the GCE for pisoCentralFoam solver (table layout)
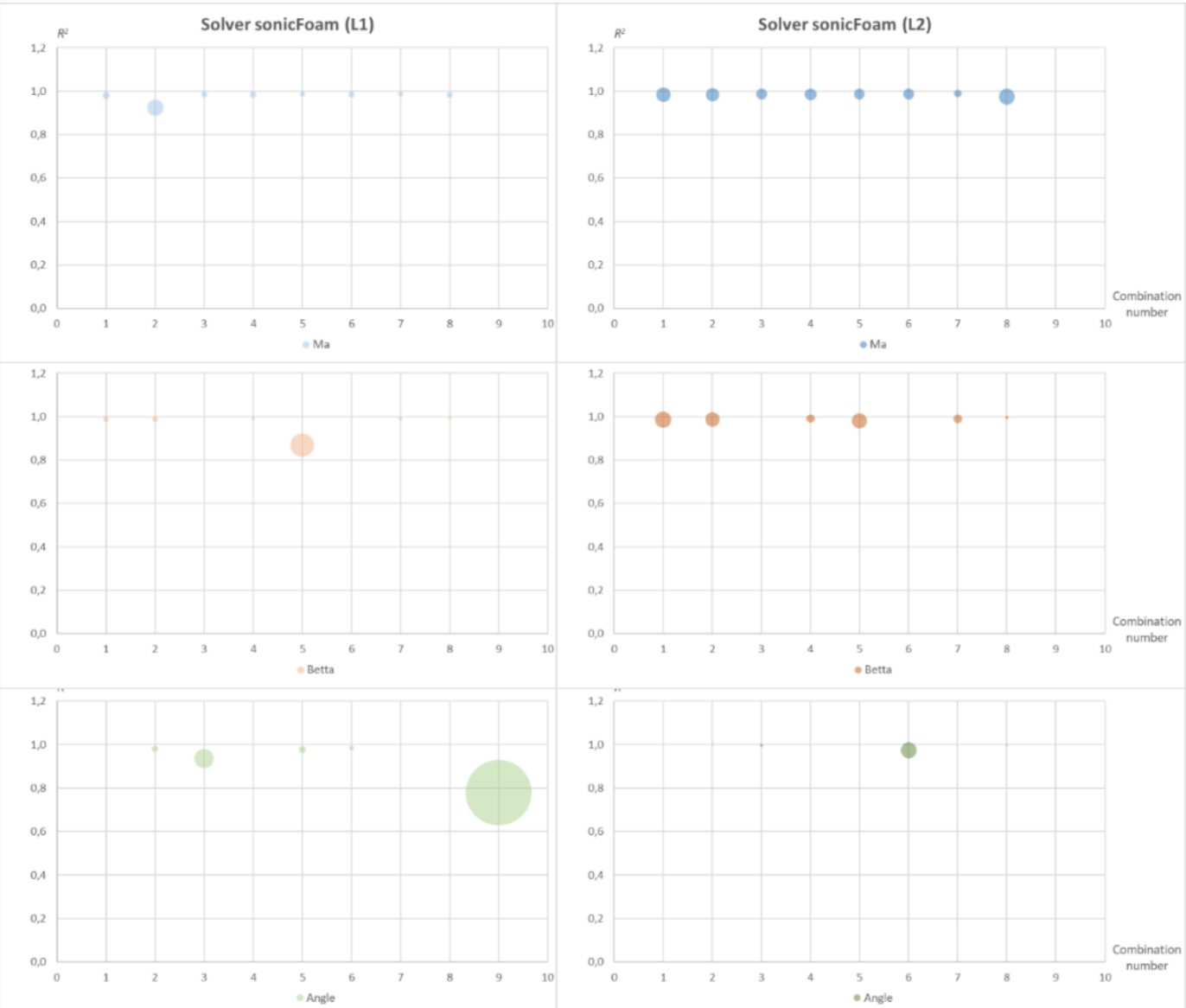

Fig. 11. Visual map of the GCE for sonicFoam solver (table layout) 


\section{Discussion of Experimental Results}

Analyzing the obtained visual images, it can be noted that, in most cases, the curve shapes for norms L1 and L2 are similar for a fixed value of the input parameter and the corresponding combinations of the remaining input parameters. However, in some cases deviations are observed. In particular, for sonicFoam solver (Fig. 5) with a fixed input parameter - Mach number and cone half-angle $-10^{\circ}$ and angle of attack $-5^{\circ}$, a significantly greater curvature of the approximating curve for the L1 norm is observed, as well as the osculation of curves for the L1 and L2 norms. Similar anomalies were also observed for the same solver and the L1 norm in two other cases:

1. Fixed input parameter - angle of attack, Mach number -5 , half-angle $-10^{\circ}$.

2. Fixed input parameter - half-angle, Mach number -5 , and angle of attack $-5^{\circ}$.

These anomalies suggest that at Mach number of 5 , half-angle of $10^{\circ}$, and angle of attack of $5^{\circ}$ for sonicFoam solver, an error could have been made in the computational experiment or in the calculation of the L1 norm. Further clarification of this fact by the authors of the computational experiment confirmed the presence of a technical error related to tabulating the results of the computational experiments for the given combination of input parameters the corresponding value of the L2 norm fell into the table cell for the L1 norm by mistake.

Analyzing the visual map for solvers (Fig. 6-8), it can also be noticed that for:

- 2 cases for rhoCentralFoam the applied approximation methods give rather different results in terms of flow ( 2 large bubbles) and all of them are for the L1 norm and the angle of attack as fixed parameter, which may mean that some approximation methods are not suitable in this case.

- 4 cases for pisoCentralFoam the applied approximation methods give rather different results in terms of flow ( 4 large bubbles) and all of them are for the L1 norm ( 3 of them - for the angle of attack as fixed parameter and one - for the half-angle as fixed parameter). This also may mean that some approximation methods are not suitable in this case.

- 3 cases for sonicFoam the applied approximation methods give rather different results in terms of flow (3 large bubbles) and all of them are for the L1 norm. Moreover, for the sonicFoam solver, this corresponds to the following situations:

1. Fixed input parameter - Mach number, half-angle $-10^{\circ}$, angle of attack $-5^{\circ}$.

2. Fixed input parameter - half-angle, Mach number -5 , angle of attack $-5^{\circ}$.

3. Fixed input parameter - angle of attack, Mach number -7 , half-angle $-20^{\circ}$.

The first two cases correspond to an already discovered problem by visualizing the approximating curves. The third case is typical for other computational experiments and may indicate the need for an additional series of experiments for intermediate values of the corresponding parameters in order to clarify the nature of the dependence and, possibly, to correct the list of types of approximating curves for sonicFoam solver.

\section{Conclusion}

The paper considers the problem of evaluating the state of a generalized computational experiment and the methods for construct visual maps of a GCE it visual analysis. An approach is proposed to construct visual maps of GCE based on the sequential applying of two methods: visualization of a series of dependencies of the output simulation parameters on the input ones for a given set of approximating functions and visualization of approximation parameters for different value ranges of the input parameters with different layout methods.

Due to the use of the proposed construct visual maps methods, it was been possible to identify experiments that have signs of errors as well as the value ranges of the input parameters for which it is advisable to conduct additional experiments for intermediate values. These circumstances make it possible to correct the further plan of conducting computational experiments.

In combination with other methods for verifying GCE data [12], the presented methods can be of great help to researchers in planning and performing computational experiments. 
Their software implementation will allow creating a reliable and efficient visualization tool that can be used in a wide range GCEs.

It should also be noted that application of the above visual map constructing methods could only be possible with a sufficient number of already performed computational experiments within a GCE, since to construct the approximating curves, at least 2 points are required with a fixed input parameter.

\section{Acknowledgements}

The research has been supported by Russian Science Foundation (project No. 18-1100215).

\section{References}

1. Bondarev, A.E.: On the Construction of the Generalized Numerical Experiment in Fluid Dynamics. Mathematica Montisnigri, XLII, 52-64 (2018).

2. Bondarev, A.E., Galaktionov, V.A.: Generalized Computational Experiment and Visual Analysis of Multidimensional Data. Scientific Visualization, 11(4), 102-114 (2019). doi: 10.26583/sv.11.4.09

3. Alekseev, A., Bondarev, A., Galaktionov, V., Kuvshinnikov, A., Shapiro, L.: On Applying of Generalized Computational Experiment to Numerical Methods Verification. In: CEUR Workshop Proceedings of GraphiCon 2020, vol. 2744 (2020). doi: 10.51130/graphicon-2020-2-3-19

4. Bondarev, A.E., Kuvshinnikov, A.E.: Analysis of the Accuracy of OpenFOAM Solvers for the Problem of Supersonic Flow Around a Cone. In: Shi, Y. et al. (eds.) ICCS 2018, LNCS, vol. 10862. pp. 221-230. Springer, Cham (2018). doi: 10.1007/978-3-319-93713-7_18

5. Andreev, S.V., et al.: A Computational Technology for Constructing the Optimal Shape of a Power Plant Blade Assembly Taking into Account Structural Constraints. Programming and Computer Software, 43(6), 345-352 (2017). doi: 10.1134/So361768817060020

6. Gorban, A.N., Kegl, B., Wunsch, D., Zinovyev, A.Y. (eds.): Principal Manifolds for Data Visualisation and Dimension Reduction, Springer-Verlag Berlin Heidelberg (2007). doi: 10.1007/978-3-540-73750-6

7. Zakharova, A.A., Podvesovskii, A.G., Shklyar, A.V.: Visual and Cognitive Interpretation of Heterogeneous Data. In: Int. Arch. Photogramm. Remote Sens. Spatial Inf. Sci., XLII-2/W12, pp. 243-247 (2019). doi: 10.5194/isprs-archives-XLII-2-W12-243-2019

8. OpenFOAM. Free CFD Software. The OpenFOAM Foundation, https://openfoam.org, last accessed 2021/o7/12.

9. Zakharova A., Korostelyov D., Podvesovskii A.: Evaluating State Effectiveness in Control Model of a Generalized Computational Experiment. In: Kravets A.G. et. al. (eds.): Creativity in Intelligent Technologies and Data Science. CIT\&DS 2021. Communications in Computer and Information Science, Vol 1448. Springer, Cham (2021). doi: 10.1007/978-3030-87034-8_16

10. Andreev, S.V., Bondarev, A.E., Bondareva, N.A.: Stereoscopic Construction of Textual Information in Presentations of Research Results on an Autostereoscopic Monitor. Scientific Visualization, 12(1), 132-139 (2020). doi: 10.26583/sv.12.1.12

11. Baltagi, B.H.: Econometrics. 5th edn. Springer-Verlag Berlin Heidelberg (2011). doi: 10.1007/978-3-642-20059-5

12. Zakharova, A.A., Korostelyov, D.A.: Application of Visual Analytics Methods to Reduce the Dimensionality of Decision-making Problems. Scientific Visualization, 12(4), 2332 (2020). doi: 10.26583/sv.12.4.03 